\title{
THE CONVERGENCE OF SEQUENCES OF RATIONAL FUNCTIONS OF BEST APPROXIMATION. II ( $\left.{ }^{1}\right)$
}

\author{
BY \\ J. L. WALSH
}

Unless the poles of a rational function of best approximation are prescribed in advance, the determination of those poles even approximately may be difficult, yet may be necessary for a complete study of approximation or convergence. Certain cases where this determination is possible have recently been considered [1] by the present author; the object of the present note is to enlarge the category of those cases, especially to study approximation on a set having no interior points, and on a set with several components.

Montessus de Ballore considered in 1902 an analogous situation related to the Padé table, with analogous results so far as concerns regions of convergence, but his methods relate to the special properties of the Taylor development and do not apply to the hypotheses of the present paper. Here our methods are based primarily on the deeper use of geometric degree of convergence, in various forms, including methods developed [2] in the theory of approximating polynomials.

A rational function of the form

$$
\frac{a_{0} z^{j}+a_{1} z^{j-1}+\cdots+a_{j}}{b_{0} z^{k}+b_{1} z^{k-1}+\cdots+b_{k}}, \quad \sum\left|b_{i}\right| \neq 0,
$$

is said to be of type $(j, k)$, and we study sequences $(j \rightarrow \infty, k$ constant) of functions of such type with suitable degrees of convergence; our primary interest is in functions of given type of best approximation.

If $E$ is a point set whose complement $K$ is connected, and regular in the sense that there exists Green's function $G(z)$ for $K$ with pole at infinity, we denote generically by $E_{\sigma}(\sigma>1)$ the locus $\Phi(z)=\sigma$ in $K, \Phi(z) \equiv \exp [G(z)]$.

LEMmA 1. Let the rational functions $r_{n \nu}(z)$ of respective types $(n, \nu)$ satisfy the inequality

$$
\limsup _{n \rightarrow \infty}\left\|r_{n \nu}(z)\right\|^{1 / n} \leqq 1 / \rho_{1}, \quad 1<\rho_{1} \leqq \infty,
$$

where $\nu$ is constant and the norm is a pth power $(p>0)$ norm on a closed

Presented to the Society, August 15, 1963; received by the editors March 30, 1964.

( $\left.{ }^{1}\right)$ Research supported in part by the U. S. Air Force Office of Scientific Research. Abstract published in Notices of the American Mathematical Society 10 (1963) 588. 
bounded set $E$ (not a single point) whose complement is simply connected. The norm is defined as the pth root of the integral of the pth power of the modulus of $r_{n v}(z)$ over the boundary of $E$, assumed rectifiable. Suppose the finite poles of the $r_{n v}(z)$ are uniformly bounded. Let $S$ be a closed set lying in the closed interior of the locus $E_{\sigma}, 1<\sigma<\rho_{1}$, and containing no limit point of the poles of the $r_{n v}(z)$. Then the sequence $r_{n v}(z)$ converges uniformly to zero on $S$, and we have

$$
\limsup _{n \rightarrow \infty}\left[\max \left|r_{n \nu}(z)\right|, z \text { on } S\right]^{1 / n} \leqq \sigma / \rho_{1} .
$$

The $r_{n \nu}(z)$ need not be defined for every $n$.

We interpret $r_{n \nu}(z)$ as the quotient of a polynomial $P_{n}(z)$ of degree $n$ by a polynomial of degree $\nu$ having unity as the coefficient of its highest power of $z$. For $n$ sufficiently large and for $z$ on $S$, the latter polynomial has a positive lower bound $m_{1}$ independent of $n$, so we have

$$
\left|r_{n v}(z)\right| \leqq\left|P_{n}(z)\right| / m_{1}, \quad z \text { on } S .
$$

Likewise, for $z$ on $E$ and for $n$ sufficiently large there exists $M_{2}$ independent of $n$ such that we have

$$
\left|P_{n}(z)\right| / M_{2} \leqq\left|r_{n v}(z)\right|, \quad z \text { on } E \text {. }
$$

By virtue of a known lemma $[2, \S 5.2$, Lemma] we may write

$$
\left[\max \left|P_{n}(z)\right|, z \text { on } S\right] \leqq L^{\prime} \sigma^{n}\left\|P_{n}(z)\right\|,
$$

where $L^{\prime}$ is a constant independent of $n$ and the norm is $p$ th power on $E$. For the $p$ th power norms on $E$ we clearly have by (4)

$$
\left\|P_{n}(z)\right\| / M_{2} \leqq\left\|r_{n \nu}(z)\right\| \text {. }
$$

Successive application of (3), (5), (6), and (1) now yields (2).

Lemma 1 is the chief tool in the proof of

TheOREM 1. Let $E$ satisfy the conditions of Lemma 1, and let the function $f(z)$ be analytic on $E$, meromorphic with precisely $\nu$ poles interior to $E_{\rho}$. Let the rational functions $R_{n v}(z)$ of respective types $(n, \nu)$ satisfy

$$
\limsup _{n \rightarrow \infty}\left\|f(z)-R_{n v}(z)\right\|^{1 / n} \leqq 1 / \rho, \quad 1<\rho \leqq \infty,
$$

where the pth power norm $(p>0)$ is used on $E$. Let $D$ denote the interior of $E_{\rho}$ with the poles of $f(z)$ deleted. Then for $n$ sufficiently large the function $R_{n \nu}(z)$ has precisely $\nu$ finite poles, which approach, respectively, the $\nu$ poles of $f(z)$ interior to $E_{\rho}$. The functions $R_{n v}(z)$ approach $f(z)$ throughout $D$. For any closed set $S$ in $D$ and in the closed interior of $E_{\sigma}, 1<\sigma<\rho$, we have

$$
\limsup _{n \rightarrow \alpha}\left[\max \left|f(z)-R_{n \nu}(z)\right|, z \text { on } S\right]^{1 / n} \leqq \sigma / \rho .
$$


It will appear from our discussion that for $n$ sufficiently large $R_{n v}(z)$ has no poles on $E$, since the poles of $R_{n v}(z)$ approach those of $f(z)$, multiplicities included.

Let $f_{0}(z)$ denote $f(z)$ minus the sum $r_{0}(z)$ of the principal parts of the $\nu$ poles of $f(z)$ interior to $E_{\rho}$, and let $r_{n}(z)$ denote the sum of the principal parts of the finite poles of $R_{n v}(z)$. There exist $[2, \S 4.5]$ polynomials $p_{n}(z)$ of respective degrees $n-\nu$ such that we have

$$
\limsup _{n \rightarrow \infty}\left\|f_{0}(z)-p_{n}(z)\right\|^{1 / n} \leqq 1 / \rho,
$$

where the norm is as in (7). If the polynomial part of $R_{n v}(z)$ is denoted by $p_{n}(z)+q_{n}(z)$, we have $[2, \S 5.2]$

$$
\limsup _{n \rightarrow \infty}\left\|r_{0}(z)-r_{n}(z)-q_{n}(z)\right\|^{1 / n} \leqq 1 / \rho .
$$

Inequality (10) is precisely of form (1), where $n$ and $\nu$ in (1) are to be replaced by $n+\nu$ and $2 \nu$ of Theorem 1 . If $R_{n \nu}(z)$ has effectively $\nu$ finite poles, it may be written as a function $r_{n}(z)$ of type $(\nu-1, \nu)$ plus a polynomial $p_{n}(z)+q_{n}(z)$ of degree $n-\nu$; if $R_{n v}(z)$ has fewer than $\nu$ finite poles, the degree of the remaining polynomial is correspondingly increased, but not beyond $n$. We assume for the moment that the finite poles of the $R_{n v}(z)$ are uniformly bounded, so that Lemma 1 is applicable; this restriction is later to be removed.

The function $r_{0}(z)$ in (10) is independent of $n$, and we proceed to discuss the finite poles of the function approaching $r_{0}(z)$, namely, the poles of $r_{n}(z)$. Let $\alpha$ be a typical pole of $r_{0}(z)$ interior to $E_{\rho}$, and let $\nu+1$ be mutually disjoint open annuli $A_{j}$ interior to $E_{\rho}$, each with center $\alpha$, separate $\alpha$ from all zeros of $r_{0}(z)$ and from all other poles of $r_{0}(z)$. If any subsequence of the $r_{n}(z)$ is given, there exists a new subsequence having no limit point of poles in at least one annulus $A_{j}$. If the given subsequence has no limit point of poles in $A_{1}$, the conclusion is established; if it has a limit point of poles in $A_{1}$, a new subsequence has for each term at least one pole in $A_{1}$ and for the new subsequence that same limit point of poles. If the new subsequence has no limit point of poles in $A_{2}$, the conclusion is established; if it has a limit point of poles in $A_{2}$, a new subsequence of the previous subsequence has for each term at least one pole in $A_{2}$ and for the new subsequence that same limit point of poles. We continue this procedure, and since each $r_{n}(z)$ has at most $\nu$ finite poles, we must eventually reach an annulus, say $A_{v+1}$, which for $n$ sufficiently large contains no pole of some subsequence of the $r_{n}(z)$. Let $C$ be a circle whose center is $\alpha$ which lies in $A_{v+1}$. The subsequence of the $r_{n}(z)+q_{n}(z)$ converges uniformly to $r_{0}(z)$ on $C$, by Lemma 1. It follows [1, Lemma 1] that interior to $C$ and for $n$ sufficiently large the subsequence $r_{n}(z)$ must have at least as many 
poles as does $r_{0}(z)$. We can apply this discussion to each of the $\nu$ poles $\alpha$ of $r_{0}(z)$, so every subsequence of the $r_{n}(z)$ admits a new subsequence having for $n$ sufficiently large in a suitable neighborhood of each of the $\nu$ poles of $r_{0}(z)$ interior to $E_{\rho}$ (at least and therefore) precisely as many poles as does $r_{0}(z)$. Then the entire sequence $r_{n}(z)$ has this same property, of having for $n$ sufficiently large in the neighborhood of each of the $\nu$ poles of $r_{0}(z)$ interior to $E_{\rho}$ precisely as many poles as does $r_{0}(z)$, and has no other poles; that is to say, for $n$ sufficiently large $R_{n v}(z)$ has precisely $\nu$ finite poles, which approach, respectively, the $\nu$ poles of $f(z)$ interior to $E_{\rho}$. Inequality (8) follows by Lemma 1 from (10) and from the following consequence $[2, \$ 4.7]$ of $(9)$ :

$$
\limsup _{n \rightarrow \infty}\left[\max \left|f_{0}(z)-p_{n}(z)\right|, z \text { on } E_{\sigma}\right]^{1 / n} \leqq \sigma / \rho .
$$

It may be noted that we have here applied various lemmas relating to degree of convergence of polynomials, that is, to the case $\nu=0$; this turns out to be possible because $R_{n v}(z)$ has a polynomial part which largely determines the convergence properties of the whole.

We have thus far assumed without proof that the finite poles of the $R_{n v}(z)$ are uniformly bounded. If this assumption is not fulfilled, as we now suppose, Lemma 1 cannot be applied to the study of the sequence $r_{0}(z)-r_{n}(z)-q_{n}(z)$. There exists a subsequence of the latter functions, now denoted by $\psi_{n}(z)$, such that precisely $\mu$ finite poles $\alpha_{j}$ of each $\psi_{n}(z)$ become infinite as $n$ becomes infinite, $0<\mu \leqq \nu$, while the remaining poles $(2 \nu-\mu$ or fewer in number) are uniformly bounded, say in modulus less than $A$, where $E$ and the $\nu$ poles of $f(z)$ lie in the circle whose center is the origin and radius $A$. When one or more poles $\alpha_{j}$ of $\psi_{n}(z)$ are in modulus greater than $A$, say $\mu^{\prime}$ in number, the function $\psi_{n}(z)$ of the subsequence is to be replaced by

$$
\psi_{n}(z) \cdot \prod_{j=1}^{\mu^{\prime}} \frac{z-\alpha_{j}}{-\alpha_{j}} \equiv \phi_{n}(z), \quad 1 \leqq \mu^{\prime} \leqq \mu,
$$

where the $\alpha_{j}$ depend on $n$, and where $\phi_{n}(z)$ has no more than $2 \nu-\mu$ finite poles; this replacement alters neither the limit of $\psi_{n}(z)$ interior to the circle nor such a relation as (10). The discussion of the $\psi_{n}(z)$ already given commencing with (10) is now valid as well for the sequence of the modified $\psi_{n}(z)$, to which Lemma 1 applies. In some annulus $A_{v+1}$ pertaining to each pole of $r_{0}(z)$, these modified functions $\psi_{n}(z)$ or a subsequence have no poles and approach zero uniformly, so the same is true of the corresponding functions $r_{0}(z)-r_{n}(z)-q_{n}(z)$. It follows as before that the functions $r_{n}(z)$ have for $n$ sufficiently large at least $\nu$ finite poles approaching the respective poles of $r_{0}(z)$, which is impossible. This contradiction shows that the finite 
poles of the $r_{n}(z)$ are bounded, and completes the proof of Theorem 1 . To Theorem 1 we add the

Corollary. Under the conditions of Theorem 1 we have

$$
\limsup _{n \rightarrow \infty}\left[\max \left|f(z)-R_{n v}(z)\right|, z \text { on } E\right]^{1 / n} \leqq 1 / \rho .
$$

In (8) we chose $S$ as $E_{\sigma}, 1<\sigma<\rho$, where $\sigma$ is chosen so small that no pole of $f(z)$ lies on or within $E_{\sigma}$. The maximum of $\left|f(z)-R_{n v}(z)\right|$ on $E$ is not greater than the maximum on $E_{\sigma}$, so (8) follows with reference to the maximum on $E$, and letting $\sigma$ approach unity yields (12).

The particular advantage of Theorem 1 over the results of [1] is that Theorem 1 does not require $E$ to be a point set bounded by one or more analytic Jordan curves; it is sufficient if $E$ consists of a finite number of suitably connected rectifiable Jordan arcs.

Theorem 1 has further consequences. Thus, with the hypothesis of Theorem 1 the inequality

$$
\limsup _{n \rightarrow \infty}\left\|f(z)-R_{n \mu}(z)\right\|^{1 / n} \leqq 1 / \rho, \quad 1<\rho \leqq \infty,
$$

is impossible with $R_{n_{\mu}}$ of type $(n, \mu)$ and $\mu<\nu$; because by the proof of Theorem 1 , for $n$ sufficiently large the function $R_{n_{\mu}}(z)$ must have at least as many poles interior to $E_{\rho}$ as does $f(z)$.

Theorem 2. With the hypothesis of Theorem 1 on $E$ and $f(z)$, let the $R_{n \nu}(z)$ be the (i.e., any) rational functions of type $(n, v)$ of best approximation to $f(z)$ on $E$ in the sense of least pth powers, $p>0$. Then (7) and the conclusion of Theorem 1 and its corollary are valid.

It follows from [1, Theorem 2] that there exist rational functions $R_{n v}(z)$ of respective types $(n, \nu)$ which satisfy $(7)$, a fortiori, the rational functions of best approximation also satisfy (7), so Theorem 2 follows from Theorem 1 and its corollary.

TheoRem 3. Under the conditions of Theorem 2 , let $\rho(1<\rho \leqq \infty)$ be the largest number such that $f(z)$ is meromorphic with precisely $\nu$ poles interior to $E_{\rho}$. Then the equality sign holds in (7) for the extremal $R_{n v}(z)$, and also for any functions $R_{n v}(z)$ for which the inequality sign ( $\left.\leqq\right)$ holds in (7).

If the first member of (7) equals $1 / \rho_{1}(<1 / \rho)$, we may write for the $p$ th power norm on $E$

$$
\limsup _{n \rightarrow \infty}\left\|R_{n+1, \nu}(z)-R_{n \nu}(z)\right\|^{1 / n} \leqq 1 / \rho_{1} .
$$

By Theorem 1 the poles of this rational function approach those of $f(z)$, so by Lemma 1 the sequence $R_{n v}(z)$ converges uniformly, necessarily to 
$f(z)$ or its analytic extension, throughout some annular region containing $E_{\rho}$ in its interior. This contradicts the definition of $\rho$.

In Theorem 1 , its corollary and Theorem 2 , it is not essential that the $R_{n v}(z)$ be defined for every $n$, but in Theorem 3 that condition (or a substitute) is essential, because we proceed from (7) directly to an inequality for the norm of $R_{n+1, v}(z)-R_{n \nu}(z)$.

Hitherto, we have considered wholly the $p$ th power norm on $E$ in the hypothesis. If we consider the Tchebycheff (uniform) norm, $E$ may be more general in that it may consist of even an infinite number of components, and its boundary need not be rectifiable.

Let us indicate the proof of

Theorem 4. Let $E$ be a closed bounded point set whose complement is connected and regular. Let the function $f(z)$ be analytic on $E$, meromorphic with precisely $\nu$ poles interior to $E_{\rho}, 1<\rho \leqq \infty$. Let the rational functions $R_{n \nu}(z)$ of respective types $(n, \nu)$ satisfy $(7)$, where the Tchebycheff norm is used on $E$. Then for $n$ sufficiently large the function $R_{n \nu}(z)$ has precisely $\nu$ finite poles, which approach, respectively, the $\nu$ poles of $f(z)$ interior to $E_{\rho}$. If $D$ denotes the interior of $E_{\rho}$ with the $\nu$ poles of $f(z)$ deleted, the sequence $R_{n \nu}(z)$ converges to $f(z)$ throughout $D$, and for any closed set $S$ in $D$ and in the closed interior of $E_{\sigma}, 1<\sigma<\rho$, we have (8).

If the $R_{n \nu}(z)$ are the rational functions of type $(n, \nu)$ of best approximation to $f(z)$ on $E$ in the sense of Tchebycheff, then (7) is satisfied.

Thus far the $R_{n v}(z)$ need not be defined for every $n$, but below they shall be so defined.

Whether the $R_{n v}(z)$ are extremal or not, let $\rho$ be the largest number such that $f(z)$ is meromorphic with precisely $\nu$ poles interior to $E_{\rho}$. If (7) holds, then (7) holds with the equality sign.

The proof of Theorem 4 follows those of Theorems 1-3, except that in following the proof of Theorem 1, [1, Lemma 3 (slightly modified) ] is used instead of the present Lemma 1 . The possibility that the finite zeros of the $R_{n \nu}(z)$ are not bounded is treated as in the proof of Theorem 1. Details are left to the reader.

In Theorem 4 let the $R_{n \nu}(z)$ be defined for every $n$, let the $\nu$ poles $\alpha_{j}$ of $f(z)$ be ordered so that $\Phi\left(\alpha_{1}\right) \leqq \Phi\left(\alpha_{2}\right) \leqq \cdots \leqq \Phi\left(\alpha_{v}\right)$, and suppose either $\Phi\left(\alpha_{\mu}\right)<\sigma<\Phi\left(\alpha_{\mu+1}\right)$ or $\sigma<\Phi\left(\alpha_{1}\right)$ in which case we set $\mu=0$. If (8) is interpreted as involving the Tchebycheff norm on $E_{\sigma}$, where $S$ is chosen as $E_{\sigma}$, then (8) is a special case of (7) with Tchebycheff norm as in the hypothesis of Theorem 4 , where $E_{\sigma}$ of (8) takes the role of $E$ in (7), since $\left(E_{\sigma}\right)_{\rho / \sigma}=E_{\rho}$; of course, $f(z)$ has only $\nu-\mu$ poles between $E_{\sigma}$ and $E_{\rho}$, as has $R_{n v}(z)$ for $n$ sufficiently large. By Theorem 4 we conclude under these circumstances 


$$
\underset{n \rightarrow \infty}{\limsup }\left[\max \left|f(z)-R_{n \nu}(z)\right|, z \text { on } E_{\sigma}\right]^{1 / n}=\sigma / \rho
$$

this relation is valid likewise for $\Phi\left(\alpha_{v}\right)<\sigma<\rho$, as follows by the reasoning associated with the generalized Bernstein lemma [2, \$4.6], even though $R_{n v}(z)$ is not a polynomial.

We formulate also the

Corollary. With the hypothesis of the last part of Theorem 4, let $T$ be any bounded continuum (not a single point) in $D$, but having at least one point exterior to $E$. Then we have

$$
\underset{n \rightarrow \infty}{\limsup }\left[\max \left|f(z)-R_{n \nu}(z)\right|, z \text { on } T\right]^{1 / n}=[\max \Phi(z), z \text { on } T] / \rho .
$$

It is a consequence of (8) that for arbitrary $T$ of the corollary we have

$$
\limsup _{n \rightarrow \infty}\left[\max \left|f(z)-R_{n \nu}(z)\right|, z \text { on } T\right]^{1 / n} \leqq[\max \Phi(z), z \text { on } T] / \rho,
$$

so the function $G(z)-\log \rho$ is a harmonic majorant for $\left[f(z)-R_{n v}(z)\right]^{1 / n}$ in $(D-E)$, and $[4$, corollary to Theorem 1$]$ by our discussion of the case $T=E_{\sigma}$ is even an exact harmonic majorant. The corollary follows.

Under the conditions of Theorem 3, or of the latter part of Theorem 4, let (7) for Tchebycheff norm hold with the equality sign for the $R_{n v}(z)$. Then by the reasoning already mentioned $[2, \$ 4.6]$ it follows $[3,4]$ that $G(z)-\log \rho$ is an exact harmonic majorant for the sequence $\left[R_{n \nu}(z)\right]^{1 / n}$ exterior to $E_{\rho}$. On any closed set interior to $E_{\rho}$ we have uniformly $R_{n \nu}(z)$ $\rightarrow f(z)$, so $V(z) \equiv 0$ is an exact harmonic majorant for the sequence $\left[R_{n v}(z)\right]^{1 / n}$ and for every subsequence interior to $E_{\rho}$ except where $f(z) \equiv 0$. Consequently, $[3,84]$ each point $z_{0}$ on $E_{\rho}$ which is a limit point of points interior to $E_{\rho}$ on which $f(z) \not \equiv 0$, is a limit point of the zeros of $R_{n \nu}(z)$ for $n$ sufficiently large, and if $\gamma$ denotes an arbitrary circular disc with center $z_{0}$ and $N_{n}$ denotes the number of zeros of $R_{n \nu}(z)$ in $\gamma$, then we have

$$
\limsup _{n \rightarrow \infty} N_{n} / n>0 \text {. }
$$

Moreover, the sequence $R_{n \nu}(z)$. cannot converge or even be uniformly bounded on a continuum (not a single point) exterior to $E_{\rho}$.

The above results apply to a function $f(z)$ analytic on $E$ and meromorphic with precisely a finite number $\nu_{1}, \nu_{2}, \ldots$ of poles in each of a finite or infinite sequence of respective regions $E_{\rho_{1}}, E_{\rho_{2}}, \cdots$; indeed, $f(z)$ may be meromorphic at every finite point of the plane. The extremal functions $R_{n \nu}(z)$ already studied correspond to the functions of the respective $\left(\nu_{j}+1\right)$ st rows of the analogue defined in [1] of the Padé table. However, if two poles $\alpha_{\mu}$ and $\alpha_{\mu+1}$ of $f(z)$ lie on $E_{\sigma}, 1<\sigma<\rho$, where the poles are ordered so that $\Phi\left(\alpha_{1}\right) \leqq \Phi\left(\alpha_{2}\right) \leqq \cdots$, then the present results do not discuss the behavior 
of $R_{n_{\mu}}(z)$. Nevertheless, it is a consequence of the method of proof of Theorem 1 that, for instance, with $\Phi\left(\alpha_{\mu}\right)=\Phi\left(\alpha_{\mu+1}\right)<\Phi\left(\alpha_{\mu+2}\right)$, the following inequality is not possible with $\Phi\left(\alpha_{\mu+2}\right)=\rho$ :

$$
\limsup _{n \rightarrow \infty}\left[\max \left|f(z)-R_{n \mu}(z)\right|, z \text { on } E\right]^{1 / n} \leqq 1 / \rho .
$$

More generally, it may be noted by the method of proof of Theorem 1 that inequality (7) for the Tchebycheff norm, valid for some $f(z)$, known merely to be analytic on $E$ and meromorphic interior to $E_{\rho}$, implies that $f(z)$ has no more than $\nu$ poles interior to $E_{\rho}$.

In this connection the following theorem is worth formulating by way of contrast:

Theorem 5. Let $E$ and $E_{\sigma}$ be as in Lemma 1 , and suppose $\rho(<\infty)$ is the largest number such that $f(z)$ is meromorphic with precisely $\nu$ poles $(\nu \geqq 0)$ in the interior of $E_{\rho}$. Let the sequence of points $\alpha_{1}, \alpha_{2}, \alpha_{3} \cdots$ not on $E$ be given; these points may be chosen everywhere dense interior to $E_{\rho}$ (but not on $E)$, or in the complement of $E$. Then there exist rational functions $R_{n, v+1}(z)$ of respective types $(n, \nu+1)$ each with a single pole in $\alpha_{n}$ such that there is valid

$$
\limsup _{n \rightarrow \infty}\left[\max \left|f(z)-R_{n, v+1}(z)\right|, z \text { on } E\right]^{1 / n}=1 / \rho .
$$

It may be noted that for no functions $R_{n, v+1}(z)$ of types $(n, \nu+1)$ can this equation become a strong inequality $(<)$ unless $f(z)$ is meromorphic with precisely one pole on $E_{\rho}$.

There exist (for instance the extremal functions of Theorem 4) rational functions $r_{n v}(z)$ of respective types $(n, \nu)$ such that we have

$$
\limsup _{n \rightarrow \infty}\left[\max \left|f(z)-r_{n \nu}(z)\right|, z \text { on } E\right]^{1 / n}=1 / \rho .
$$

To prove Theorem 5 we need merely set $R_{n, v+1}(z) \equiv r_{n-1, v}(z)+A_{n} /\left(z-\alpha_{n}\right)$, $n \geqq \nu$, where the $A_{n}(>0)$ are chosen so small that each $R_{n, v+1}(z)$ has effectively a pole in $\alpha_{n}$, and so that we have

$$
\limsup _{n \rightarrow \infty}\left[\max \left|A_{n} /\left(z-\alpha_{n}\right)\right|, z \text { on } E\right]^{1 / n}<1 / \rho .
$$

Theorem 5 suggests the difficulty of locating the poles of the extremal $R_{n, \nu+1}(z)$ when there exists no $E_{\rho}$ whose interior contains precisely $\nu+1$ poles of $f(z)$. However, under the conditions of Theorem 5 each pole of $f(z)$ interior to $E_{\rho}$ is a limit of poles of the $R_{n, v+1}(z)$. In fact we have

THEOREM 6. With the hypothesis of the last part of Theorem 4, suppose $f(z)$ is not meromorphic with fewer than $\mu-\nu+1$ poles on $E_{\rho}$; let the $R_{n, \mu}(z)$ be the rational functions of type $(n, \mu)$ of best approximation with Tchebycheff norm to $f(z)$ on $E, \mu>\nu$. Then we have 


$$
\limsup _{n \rightarrow \infty}\left[\max \left|f(z)-R_{n \mu}(z)\right|, z \text { on } E\right]^{1 / n} \leqq 1 / \rho,
$$

and each pole of $f(z)$ interior to $E_{\rho}$ is a limit point of poles of the $R_{n_{\mu}}(z)$, possibly of greater multiplicity,

The first part of Theorem 6 follows by comparison from (7) for the extremal $R_{n v}(z)$, since the latter functions are also functions of type $(n, \mu)$; the latter part of Theorem 6 follows by the method of proof of Theorem 1 ; it is essential here to note that for any subsequence of the $R_{n \mu}(z)$ no more than $\mu-\nu$ poles of the $R_{n \mu}(z)$ can become infinite. Theorem 6 refers to the behavior of the functions in the $(\mu+1)$ st row of the analogue of the Padé table.

A result somewhat similar to Theorem 6 is

THEOREM 7. With the hypothesis of the last part of Theorem 4 we have

$$
\limsup _{n \rightarrow \infty}\left[\max \left|f(z)-R_{n n}(z)\right|, z \text { on } E\right]^{1 / n} \leqq 1 / \rho .
$$

This inequality follows at once from (7) and [1, inequality (4)]. However, it is not true that the inequality is always an equality; as a counterexample we choose $E$ arbitrarily, and $f(z)$ having as its only singularity in the extended plane an essential singularity $\alpha_{0}$ on $E_{\rho}, \rho<\infty$. Transformation of the plane by the linear transformation $z^{\prime}=1 /\left(z-\alpha_{0}\right)$ and use of the Taylor development shows that we have

$$
\limsup _{n \rightarrow \infty}\left[\max \left|f(z)-R_{n n}(z)\right|, z \text { on } E\right]^{1 / n}=0 .
$$

Compare here [5] and [6].

Although Theorem 4 applies to a set $E$ not necessarily connected, that is not true of Lemma 1 and Theorems 1-3. We proceed to discuss accordingly possible modifications of the latter.

Lemma 2. Let Lemma 1 be modified so that $E$ is a closed bounded set whose complement is connected of finite connectivity, and that each component of $E$ consists of more than one point and has a rectifiable boundary. Then the conclusion (2) persists.

The norm on $E$ is taken as the $p$ th root of the sum of integrals of $p$ th powers over the boundaries of the respective components of $E$.

Let the components of $E$ be $E^{\prime}, E^{\prime \prime}, \ldots, E^{(j)}, \ldots$, and let $\epsilon$ be chosen, $1<\epsilon<\rho_{1} / \sigma$. Let $r_{n v}(z)$ be any sequence of the given rational functions. There exists a subsequence which has no limit point of poles on some $E_{\epsilon_{1}}^{\prime}, 1<\epsilon_{1}<\epsilon$, (notation used in Lemma 1) a further subsequence having no limit point of poles on some $E_{\epsilon_{2}}^{\prime \prime}, 1<\epsilon_{2}<\epsilon$, etc.; this follows by the method of the proof of Theorem 1 concerning the $A_{j}$. We define $P_{n}(z)$ as 
in the proof of Lemma 1, so that (3) and (4) follow. Then [2, \$5.2, Lemma] yields for each $E_{t j}^{(j)}$ (instead of $S$ ) an analogue of (5), whence

$$
\left[\max \left|P_{n}(z)\right|, z \text { on } E_{0}\right] \leqq L^{\prime} \epsilon^{n}\left\|P_{n}(z)\right\|, \quad E_{0}=\sum E_{c j}^{(j)} .
$$

The first member of (13) is greater than or equal to $\left[\max \left|P_{n}(z)\right|, z\right.$ on $\left.E\right]$, so the generalized Bernstein lemma $[2, \$ 4.6]$ gives by (13)

$$
\begin{aligned}
{\left[\max \left|P_{n}(z)\right|, z \text { on } S\right] } & \leqq \sigma^{n}\left[\max \left|P_{n}(z)\right|, z \text { on } E\right] \\
& \leqq L^{\prime} \epsilon^{n} \sigma^{n}\left\|P_{n}(z)\right\| .
\end{aligned}
$$

Successive application of (3), (14), (6), and (1) yields

$$
\limsup _{n \rightarrow \infty}\left[\max \left|r_{n \nu}(z)\right|, z \text { on } S\right]^{1 / n} \leqq \epsilon \sigma / \rho_{1} \text {. }
$$

We can allow $\epsilon$ in (15) to approach unity, and have then shown that every sequence of the $r_{n v}(z)$ admits a subsequence which satisfies (2). Consequently, (2) is satisfied for the entire given sequence $r_{n \nu}(z)$. We have by use of Lemma 2 instead of Lemma 1

THEOREM 8. Theorems 1 (with corollary), 2, and 3 are valid if the hypotheses are modified so that $E$ satisfies the conditions of Lemma 2 rather than Lemma 1.

Other measures of approximation than those of $p$ th powers over a rectifiable boundary and of Tchebycheff may be considered. First, if $E$ consists (for simplicity) of a finite number of mutually exterior Jordan regions, we may measure approximation by a surface integral of $p$ th powers $(p>0)$ over $E$, as in $[2, \S 5.3]$. The analogue of Lemma 2 follows, as do the analogues of Theorems 1 (with corollary), 2, and 3. Second, if $E$ is closed and bounded with a connected complement, and if $E$ consists of a finite number of components each of which consists of more than a single point, we may measure approximation by the $p$ th $(p>0)$ root of the sum of integrals of $p$ th powers over $\gamma$ after conformal mapping of the complement of each component onto the exterior of the unit circle $\gamma$. This measure applies to very general point sets; it is used in $[2,85.4]$, and also leads to precise analogues of Lemma 2 and Theorems 1 (with corollary), 2, and 3.

Various kinds of weight functions can be introduced in these new as well as the former measures of approximation without altering the conclusions, as in $[2, \$ 5.7]$.

\section{REFERENCES}

1. J. L. Walsh, The convergence of sequences of rational functions of best approximation, Math. Ann. 155 (1964), 252-264.

2. , Interpolation and approximation, Amer. Math. Soc. Colloq. Publ., Vol. 20 Amer. Math. Soc., Providence, R. I., 1935. 
3. The analogue for maximally convergent polynomials of Jentzsch's theorem, Duke Math. J. 26 (1959), 605-616.

4. Overconvergence, degree of convergence, and zeros of sequences of analytic functions, Duke Math. J. 13 (1946), 195-234.

5.

On the overconvergence of certain sequences of rational functions of best approximation, Acta Math. 57 (1931), 411-435.

6. V. Erohin, On the best approximation of analytic functions by rational functions with free poles, Dokl. Akad. Nauk SSSR 128 (1959), 29-32.

HaRVARd UNIVERSITY,

Cambridge, Massachusetts 